Pacific Journal of Mathematics

LATTICE PROJECTIONS ON CONTINUOUS FUNCTION 


\title{
LATTICE PROJECTIONS ON CONTINUOUS FUNCTION SPACES
}

\author{
Emily Mann PeCK
}

Suppose that $X$ is a compact Hausdorff space and that $F$ is a closed (linear) sublattice of $C(X)$. We characterize those sublattices $F$ that are the ranges of (linear) lattice projections on $C(X)$ : there is a lattice projection of $C(X)$ onto $F$ if and only if there is a closed subset $Y$ of $X$ such that $F$ is lattice isomorphic to $C(Y)$ under the restriction mapping $\left.f \rightarrow f\right|_{Y}(f \in F)$. Examples are given to show that this theorem cannot be substantially improved without imposing additional conditions either on $X$ or on the sublattice $F$. If $X$ is a stonian space, then a closed sublattice $F$ of $C(X)$ is the range of a lattice projection exactly when it is the range of a positive projection.

1. Preliminary results and notation. Recall that an $(A M)$-space $E$ is a Banach lattice such that $\|x \vee y\|=\|x\| \vee\|y\|$ for each nonnegative $x$ and $y$ in $E$. Kakutani [6] proved that the (AM)-spaces are isometrically characterized as the closed sublattices of spaces $C(X)$, for $X$ a compact Hausdorff space. An order unit $e$ in $E$ is a nonnegative element of $E$ such that for each $x \geqq 0$ in $E$ there exists a real number $\lambda>0$ such that $x \leqq \lambda e . \quad E$ is an $(A M)$-space with unit if $E$ has an order unit $e$ such that the order interval $[-e, e]$ coincides with the unit ball in $E$. The weak* topology on the dual $E^{\prime}$ of $E$ will be denoted by $\sigma\left(E^{\prime}, E\right)$.

Let $X$ be a compact Hausdorff space. $\quad C(X)$ is the Banach lattice of real-valued continuous functions on $X$. For each $x \in X, \varepsilon_{x}$ is the point evaluation at $x$. The function that is identically one on $X$ will be denoted by $1_{X}$. For any subset $Y$ of $X$, define

$$
\operatorname{hull}(Y)=\{f \in C(X): f(Y)=\{0\}\}
$$

Definition 1.1. Suppose that $P$ is a projection of $C(X)$ onto a sublattice $F$ of $C(X) . \quad P$ is called an $\ell$-projection (or lattice projection) if $P$ is a linear lattice homomorphism.

Ker $P$ denotes the null space of a projection $P$. Note that $\operatorname{Ker} P$ is necessarily a lattice ideal of $C(X)$ whenever $P$ is an $\ell$-projection.

Suppose that $X$ is a compact Hausdorff space, $F$ is a closed (linear) sublattice of $C(X)$, and $P$ is a positive projection of $C(X)$ onto $F$. Then $e=P\left(1_{X}\right)$ is an order unit for $F$, and $F$ can be renormed with an 
equivalent norm $\|\cdot\|_{e}$ to be an $(A M)$-space with unit. The norm $\|\cdot\|_{e}$ is defined by

$$
\|f\|_{e}=\inf \{\lambda>0:|f| \leqq \lambda e\}, \quad f \in F .
$$

If $\|P\|=1$, then $e=P\left(1_{X}\right)$ is a unit for $F$ with the supremum norm.

There is a compact Hausdorff space $K$ such that $C(K)$ is lattice and linearly isometric to $F$ with the norm $\|\cdot\|_{e}$. In fact, $K$ can be taken to be the set of extreme points of the positive face of the dual unit ball for the norm $\|\cdot\|_{e}$ on $F$. We shall denote this particular space $K$ by $\Omega_{e}$. Equipped with the $\sigma\left(F^{\prime}, F\right)$ topology, $\Omega_{e}$ is a compact Hausdorff space. Moreover, the isometry is given by the evaluation:

$$
\begin{aligned}
& \Phi: F \rightarrow C\left(\Omega_{e}\right) \\
& \Phi(f)(\mu)=\mu(f) \text { for each } f \in F, \mu \in \Omega_{e}
\end{aligned}
$$

(see [12], p. 247). $\Phi$ is an isometry defined on $F$ with the supremum norm if and only if $\|P\|=1$.

Throughout the paper the linear structure of $C(X)$, sublattices $F$, and projection $P$ will be assumed and used even though the linear structure will not be specifically referred to each time.

The following result can be proved in a manner analogous to Corollary 4, p. 691, of [13]:

Proposition 1.2. Let $X$ be a compact Hausdorff space, and let $F$ be a closed sublattice of $C(X)$. If $P$ is a projection of norm one of $C(X)$ onto $F$, then $P$ is positive.

2. $\ell$-projections on $C(X)$. If $X$ is a compact Hausdorff space, then the range of an $\ell$-projection on $C(X)$ is lattice isomorphic to $C(K)$ for some compact Hausdorff space $K$. Does there exist an embedding of $K$ into $X$ ? In order to answer this question affirmatively, we shall need to relate linear mappings of $C(X)$ into $C(Y)$ to point maps of $Y$ into $X$. The following theorem (see Kaplan [7]) will be a major tool:

THeOREm 2.1. Let $X$ and $Y$ be compact Hausdorff spaces and let $T: C(X) \rightarrow C(Y)$ be a lattice homomorphism with $T\left(1_{X}\right)=1_{Y}$.

(a) There is a continuous function $T^{t}: Y \rightarrow X$ defined for $y \in Y$ by $f\left(T^{t}(y)\right)=T(f)(y)$ for all $f \in C(X)$. Moreover, $T^{t}$ is one-to-one whenever $T$ is onto, and $T^{t}$ is onto whenever $T$ is one-to-one.

(b) Given a continuous function $\varphi: Y \rightarrow X$, there is an associated 
linear mapping $\varphi^{0}: C(X) \rightarrow C(Y)$ defined for $f \in C(X)$ by $\varphi^{0}(f)(y)=$ $f(\varphi(y))$ for all $y \in Y$. $\left\|\varphi^{0}\right\|=1$, and $\varphi^{0}$ is an isometry whenever $\varphi$ is onto.

(c) $\left(T^{t}\right)^{0}=T$.

For any function $f: A \rightarrow B$, the symbol $\left.f\right|_{A_{0}}, A_{0} \subseteq A$, denotes the restriction of $f$ to the domain $A_{0}$. Suppose that $X$ is a compact Hausdorff space and that $Y$ is a closed subset of $X$. There is a restriction mapping $R_{Y}$ of $C(X)$ onto $C(Y)$ defined by $R_{Y}(f)(y)=f(y)$ for $y \in Y, f \in C(X)$. We shall also denote $R_{Y}(f)$ by $\left.f\right|_{Y}$. Throughout the paper the term "restriction mapping" will be used in this canonical sense. Note that $R_{Y}$ is a lattice homomorphism and that $R_{Y}\left(1_{X}\right)=1_{Y}$.

It will be necessary to extend real lattice homomorphisms (that is, real-valued lattice homomorphisms) defined on a closed sublattice $F$ of $C(X)$ to all of $C(X)$. Denote by $\Omega_{F}$ the set of extreme points of the positive face of the dual unit ball for the supremum norm of $F$. We remark that if $\Omega_{e}$ corresponds to a projection of norm one (as in $\S 1$ ), then $\Omega_{e}=\Omega_{F}$ since $\|f\|_{e}=\|f\|$ for each $f \in F$.

Lemma 2.2. (Extension lemma). Let $X$ be a compact Hausdorff space, and let $F$ be a closed sublattice of $C(X)$. Then any real lattice homomorphism on $F$ can be extended to a real lattice homomorphism on $C(X)$ that has the same norm.

Proof. Let $\xi: F \rightarrow \mathbf{R}$ be a nonzero lattice homomorphism (the zero homomorphism can be trivially extended to all of $C(X))$. Then $\xi /\|\xi\|$ is in $\Omega_{F}$ since $\xi$ generates an extreme ray of the cone in $F^{\prime}$ (see Schaefer [12], p. 213). An extreme point argument shows that any element of $\Omega_{F}$ has an extension to an extreme point of the positive face of the unit ball in $C(X)^{\prime}$. But the extreme points of the positive face of the unit ball in $C(X)^{\prime}$ are the point evaluations $\varepsilon_{x}, x \in X$, which are real lattice homomorphisms on $C(X)$. That the extension has the same norm is obvious.

The following lemma is well-known in the "folklore" of the literature; however, it does follow directly from the extension lemma.

LEMma 2.3. The set of real lattice homomorphisms on $C(X)$ coincides with the set of nonnegative multiples of the point evaluations $\varepsilon_{x}, x \in X$.

It will be useful to note that if $F$ is a closed sublattice of $C(X)$, and if $F$ is renormed with an equivalent $(A M)$-space norm $\|\cdot\|_{e}$ (see $\left.\S 1\right)$, then $\mu \in \Omega_{e}$ exactly when $\mu$ is a real lattice homomorphism on $F$ and $\|\mu\|_{e}=1$.

The first theorem identifies the range of an $\ell$-projection $P$ on $C(X)$ 
as $C(Y)$ for some closed subset $Y$ of $X$. Moreover, a converse to that statement is obtained. For the case that $\|P\|=1$, the portion (a) implies (b) was proved by Geba and Semadeni [4].

THEOREM 2.4. Let $X$ be a compact Hausdorff space and let $F$ be a closed sublattice of $C(X)$. The following assertions are equivalent:

(a) There is an $\ell$-projection of $C(X)$ onto $F$.

(b) There is a closed subset $Y$ of $X$ such that $F$ is lattice isomorphic to $C(Y)$ under the restriction mapping $R_{Y}$; moreover, if $e=R_{Y}^{-1}\left(1_{Y}\right)$, then $\|f\|_{e}=\left\|\left.f\right|_{Y}\right\|$ for each $f \in F$.

Also, if (a) holds, then $\operatorname{Ker} P=\operatorname{hull}(Y)$, and $Y$ is a subset of

$$
\left\{x \in X: P\left(1_{X}\right)(x)=e(x)=1\right\} \text {. }
$$

If $\|P\|=1$, then $F$ with the supremum norm is isometric to $C(Y)$.

Proof. If (b) is satisfied, then $R_{Y}^{-1} \circ R_{Y}$ is the desired $\ell$-projection.

Conversely, let $P$ be an $\ell$-projection of $C(X)$ onto $F$, and consider $\omega \in \Omega_{e}$, where $e=P\left(1_{X}\right)$ as in the discussion. Then $\omega \circ P$ is a real lattice homomorphism of $C(X)$ onto $\mathbf{R}$; hence by 2.3 there is an $x(\omega) \in X$ and a constant $k(\omega) \geq 0$ such that $\omega \circ P(f)=k(\omega) f(x(\omega))$ for each $f \in C(X)$ (that is, $\left.\omega \circ P=k(\omega) \varepsilon_{x(\omega)}\right)$. Consider the function

$$
\psi: \Omega_{e} \rightarrow X, \quad \text { defined by } \psi(\omega)=x(\omega), \text { for } \omega \in \Omega_{e},
$$

as given above. Then $\psi$ is a one-to-one continuous function of $\Omega_{e}$ with the $\sigma\left(F^{\prime}, F\right)$ topology into $X$. Moreover, the function $\lambda: \Omega_{e} \rightarrow \mathbf{R}$, defined by $\lambda(\omega)=k(\omega)$, for $\omega \in \Omega_{e}$, as given above, is a continuous function. Denote by $Y$ the range of $\psi$. Then $Y$ is homeomorphic to $\Omega_{e}$ via $\psi$, and so $F$ with the norm $\|\cdot\|_{e}\left(\right.$ or $\left.C\left(\Omega_{e}\right)\right)$ is lattice isometric to $C(Y)$.

It remains to show that $T: F \rightarrow C(Y)$ given by $T=\left(\psi^{-1}\right)^{t} \circ \Phi$ is the restriction mapping $R_{Y}$. Let $f \in F$, and $y \in Y$. Then

$$
\left(\psi^{-1}\right)^{t} \circ \Phi(f)(y)=\Phi(f)\left(\psi^{-1}(y)\right)=\psi^{-1}(y)(f)=f(y)=R_{Y}(f)(y) .
$$

It is clear that $e=P\left(1_{X}\right)=R_{Y}^{-1}\left(1_{Y}\right)$, and that $\|f\|_{e}=\left\|\left.f\right|_{Y}\right\|$ for each $f \in F$.

Note that $f \in \operatorname{hull}(Y)$ exactly when $f(Y)=0$; this happens exactly when $\operatorname{Pf}(Y)=0$, or when $f \in \operatorname{Ker} P$. Hence hull $(Y)=\operatorname{Ker} P$. Also, $P\left(1_{X}\right)(y)=1_{X}(y)=1$ for each $y \in Y$, so we have

$$
Y \subseteq\left\{x \in X: P\left(1_{X}\right)(x)=e(x)=1\right\} .
$$

Recall that if $Y$ is a closed subset of a compact Hausdorff space $X$, a simultaneous extension of $C(Y)$ is a bounded linear mapping 
$E: C(Y) \rightarrow C(X)$ with the property that $E(f)(y)=f(y)$ for all $y \in Y$ and $f \in C(Y)$. Dean [2] proved that the existence of a linear extension operator $E$ is equivalent to the existence of a (bounded) projection of $C(X)$ onto hull $(Y)$. The preceding proposition can be reformulated in terms of simultaneous extensions that are lattice homomorphisms as follows:

COROLlary 2.5. Let $X$ be a compact Hausdorff space, and let $F$ be a closed sublattice of $C(X)$. If $P$ is an $\ell$-projection of $C(X)$ onto $F$ with $\operatorname{Ker} P=\operatorname{hull}(Y)$, then there is a simultaneous extension operator $E: C(Y) \rightarrow C(X)$ with $\|E\|=\|P\|$ and $E$ a lattice homomorphism. Conversely, if there is a simultaneous extension operator $E: C(Y) \rightarrow C(X)$ that is a lattice homomorphism, then there is an $\ell$-projection $P$ of $C(X)$ onto a sublattice of $C(X)$ that is lattice isomorphic to $C(Y)$. Moreover, $\|P\|=\|E\|$.

Proof. If $P$ exists, then $R_{Y}^{-1}$ is the desired simultaneous extension operator. Conversely, if $E$ exists, then $E \circ R_{Y}$ is an $\ell$-projection onto a sublattice of $C(X)$ that is lattice isomorphic to $F$.

Suppose that $F$ is a closed sublattice of $C(X)$ such that $F$ is lattice isometric to the range of an $\ell$-projection of norm one on $C(X)$. Is $F$ the range of an $\ell$-projection on $C(X)$ ? If $X$ is stonian, then there is an $\ell$-projection of $C(X)$ onto $F$ as we shall see in Theorem 3.1. However, if $X$ is not stonian, it is possible for a sublattice to be lattice isometric to the range of an $\ell$-projection on $C(X)$ without itself being the range of an $\ell$-projection. In fact, the restriction that $X$ be stonian cannot even be relaxed to the restriction that $X$ be totally disconnected. Before considering such an example, we construct an $\ell$-projection of norm one associated with a given retraction.

Proposition 2.6. Let $X$ be a compact Hausdorff space and let $Y$ be a closed subset of $X$. If $r: X \rightarrow Y$ is a retraction, then there is an $\ell$-projection of norm one of $C(X)$ onto a sublattice of $C(X)$ that is lattice isometric to $C(Y)$.

Proof. Consider $r^{0}: C(Y) \rightarrow C(X)$. A simple computation shows that $r^{0} \circ R_{Y}$ is an $\ell$-projection of $C(X)$ onto $F=r^{0} \circ R_{Y}(C(X))$. Moreover, $\operatorname{Ker} r^{0} \circ R_{Y}=\operatorname{hull}(Y)$, and $R_{Y}^{-1}$ is a lattice isometry of $C(Y)$ onto $F$. Thus $\left\|r^{0} \circ R_{Y}\right\|=1$.

EXAMPLE 2.7. (See Arens [1]) We give an example of a totally disconnected compact Hausdorff space $X$ and a sublattice $F$ of $C(X)$ that is lattice isometric to $C(Y)$, where $Y$ is a retract of $X$, yet $F$ is not 
the range of any $\ell$-projection (not necessarily of norm one) on $C(X)$. By 2.6, $F$ is lattice isometric to the range of an $\ell$-projection of norm one on $C(X)$.

Let

$$
\begin{aligned}
X_{0} & =\left\{\left(\frac{1}{m}, \frac{1}{n}\right): m, n \in N, \quad m \geqq n\right\}, \\
Y & =\left\{\left(0, \frac{1}{n}\right): n \in N\right\} \cup\{(0,0)\}, \\
X & =X_{0} \cup Y .
\end{aligned}
$$

First observe that $r: X \rightarrow Y$, defined by

$$
r(t, s)=(0, s), \quad t=0,1, \frac{1}{2}, \cdots, \quad s=0,1, \frac{1}{2}, \cdots,
$$

is a retraction of $X$ onto $Y$. Then $r$ induces the $\ell$-projection $r^{0} \circ R_{Y}$ of norm one of $C(X)$ onto a sublattice of $C(X)$ that is lattice isometric to $C(Y)$.

Let $F$ be the sublattice of $C(X)$ consisting of those functions constant on $Y$. There is no $\ell$-projection of $C(X)$ onto $F$; however, $F$ is lattice isometric to $C(Y)$. That there is no projection of norm one of $C(X)$ onto $F$ is exactly the point of Arens' example. To see that there is not even a positive projection of $C(X)$ onto $F$, note that since $1_{X} \in F$, the norm of any positive projection must be one; hence Arens' proof applies, and there is no positive projection of $C(X)$ onto $F$.

It remains to show that $F$ is lattice isometric to $C(Y)$. First note that $F$ is lattice isometric to $C\left(\hat{X}_{0}\right)$, where $\hat{X}_{0}$ is the one-point compactification of $X_{0}$, via the mapping

$$
\begin{aligned}
& T: F \rightarrow C\left(\hat{X}_{0}\right), \\
& T(f)(x)=\left\{\begin{array}{cc}
f(x) & x \in X_{0} \\
f((0,0)) & x=\infty
\end{array}, \quad f \in F .\right.
\end{aligned}
$$

Hence $F$ is lattice isometric to (c), the space of convergent sequences. Clearly, $C(Y)$ is also lattice isometric to (c), and hence $F$ is lattice isometric to $C(Y)$.

We know that a norm one projection of $C(X)$ onto a sublattice of $C(X)$ is necessarily positive. It might be conjectured that a sublattice that is the range of a projection of norm one on $C(X)$ is the range of an $\ell$-projection on $C(X)$. We shall see in $\S 3$ that if $X$ is a stonian space, then the conjecture is true; however, if $X$ is a compact Hausdorff space 
that is not stonian, we claim the conjecture is not necessarily true. We are indebted to $\mathrm{H}$. Lotz for suggesting the following example that is much simpler than the one we had used in a preliminary draft.

EXAmPle 2.8. We give an example of a compact Hausdorff space $X$ and a closed sublattice $F$ of $C(X)$ such that $F$ is the range of a norm one (therefore, positive) projection on $C(X)$ and $F$ is lattice isometric to the range of an $\ell$-projection of norm one on $C(X)$, but such that $F$ is not the range of any $\ell$-projection on $C(X)$.

Let $X$ be the unit circle in the complex plane, and let $F$ consist of all the even functions in $C(X)$ :

$$
F=\{f \in C(X): f(-z)=f(z) \text { for each } z \in X\}
$$

Then $P: C(X) \rightarrow F$, defined by $P(f)(z)=(f(z)+f(-z)) / 2$, for $f \in C(X)$ and $z \in X$, is a projection of norm one of $C(X)$ onto $F$. Observe that $F$ is lattice isometric to $C(X)$, which is the range of an $\ell$-projection on $C(X)$ (the identity mapping).

If there were an $\ell$-projection of $C(X)$ onto $F$, since $F$ is lattice isometric to $C(X)$, there would be an embedding $\sigma$ of $X$ into $X$ such that $\sigma(X)$ is a closed connected subset of $X$ (an arc segment), and such that $F$ is isomorphic to $C(\sigma(X))$ via $R_{\sigma(X)}$. It is easy to see that at least one, but not both, of the elements $z$ and $-z$ for each $z \in X$ must be in $\sigma(X)$ in order that $R_{\sigma(X)}$ is one-to-one and onto. Clearly, no such arc segment exists; therefore, there is no $\ell$-projection of $C(X)$ onto $F$.

3. Projections on $C(X)$ for $X$ stonian. In this section we shall restrict our attention to projections on $C(X)$ for stonian spaces $X$. Much of the pathology encountered in the previous section cannot occur in the presence of this restriction on $X$. For example, we shall show that it is not possible for a sublattice $F$ of $C(X)$ to be lattice isomorphic to the range of an $\ell$-projection on $C(X)$ and not be the range of any $\ell$-projection on $C(X)$. (Contrast this with the situation in Example 2.7.)

Suppose that $X$ is a compact Hausdorff space. Recall that $X$ is a stonian space if the closure of each open set is open. Nakano (see [11]) proved that $C(X)$ is an order complete vector lattice if and only if $X$ is stonian. It can be shown that a sublattice that is the range of a positive projection defined on an order complete vector lattice must also be order complete as a vector lattice. Now let $X$ be a stonian space, and let $P$ be a positive projection on $C(X)$ onto a closed sublattice $F$ of $C(X)$; since $X$ is stonian, $F$ must be order complete, and the lattice isometry of $F$ with $C\left(\Omega_{e}\right)$ then shows that $\Omega_{e}$ must be stonian.

We shall state the main results for sublattices that are the ranges of 
positive projections on $C(X)$, for $X$ a stonian space, before proving any of the results.

THEOREM 3.1. Let $X$ be a stonian space and let $F$ be a closed sublattice of $C(X)$. The following are equivalent:

(a) There is a projection of norm one of $C(X)$ onto $F$.

(b) $F$ is an order complete (AM)-space with unit.

(c) $F$ is lattice isometric to a space $C(K)$, for some stonian space $K$.

(d) $F$ is lattice isometric to $C(Y)$, for some closed subset $Y$ of $X$, under the restriction mapping $R_{Y}$.

(e) There is an $\ell$-projection of norm one of $C(X)$ onto $F$.

As a corollary, we have the following result:

Corollary 3.2. Let $X$ be a stonian space, and let $Z$ be a stonian space such that $C(X)^{\prime \prime}$ is lattice isometric to $C(Z)$. Then

(a) there is an embedding of $X$ into $Z$, and

(b) there is an $\ell$-projection $P$ of norm one of $C(Z)$ onto $\varphi(C(X))$, where $\varphi$ is the canonical embedding of $C(X)$ into $C(Z)$.

If we replace the requirement that $F$ be the range of a projection of norm one by the requirement that $F$ be the range of a positive projection, then a similar theorem may be obtained by replacing "isometry" with "isomorphism" throughout 3.1. However, the proof of 3.3 will not follow exactly as that for 3.1 .

THEOREM 3.3. Let $X$ be a stonian space and let $F$ be a closed sublattice of $C(X)$. The following are equivalent:

(a) There is a positive projection of $C(X)$ onto $F$.

(b) $F$ is an order complete (AM)-space with an order unit.

(c) $F$ is lattice isomorphic to $C(K)$, for some stonian space $K$.

(d) $F$ is lattice isomorphic to $C(Y)$, for some closed subset $Y$ of $X$, under the restriction mapping $R_{Y}$.

(e) There is an $\ell$-projection of $C(X)$ onto $F$.

Note. In (b), the order interval $[-e, e]$, where $e$ is an order unit for $F$, is not in general the unit ball for $F$.

Theorems 3.1 and 3.3 are not equivalent. We obtain conditions for a closed sublattice $F$ of $C(X)$ ( $X$ stonian) that is the range of a positive projection to be (in addition) the range of a projection of norm one. We shall then be able to construct a simple example of a stonian space $X$ and a closed sublattice $F$ of $C(X)$ satisfying the conditions of Theorem 3.3 but not Theorem 3.1. 
Theorem 3.4. Let $X$ be a stonian space, and let $F$ be a closed sublattice of $C(X)$ that is the range of a positive projection on $C(X)$. The following are equivalent:

(a) There is a projection of norm one of $C(X)$ onto $F$.

(b) $F$ is lattice isometric to $C(K)$, for some stonian space $K$.

(c) $\Omega_{F}$ is $\sigma\left(F^{\prime}, F\right)$ closed.

(d) If $\left\{x_{\alpha}\right\}$ is a net in $X$ and $x$ an element of $X$ such that $\left\{\left.\varepsilon_{x_{\alpha}}\right|_{F}\right\}$ converges to $\left.\varepsilon_{x}\right|_{F}$ for $\sigma\left(F^{\prime}, F\right)$, then $\left\|\left.\varepsilon_{x_{\alpha}}\right|_{F}\right\| \rightarrow\left\|\left.\varepsilon_{x}\right|_{F}\right\|$.

(e) $Z=\left\{x\right.$ in $\left.X:\left\|\left.\varepsilon_{x}\right|_{F}\right\|=1\right\}$ is a closed subset of $X$.

(f) If $\left\{f_{\alpha}\right\}$ is an increasing net of nonnegative functions in $F$ with $\sup _{\alpha} f_{\alpha}=f$, and if $k$ is a positive constant such that $\left\|f_{\alpha}\right\| \leqq k$ for all $\alpha$, then $\|f\| \leqq k$.

(g) The unit ball in $F$ is order complete.

We shall now prove theorems 3.1, 3.3, and 3.4.

Proof of 3.1. (a) implies (b) is a consequence of 1.2; (b) implies (c) is the Kakutani representation; (d) implies (e) follows from 2.4; and (e) implies (a) is obvious. It remains to show that (c) implies (d).

Without loss of generality, we can assume that $K$ is $\Omega_{F}$. Suppose that $\Phi$ is the lattice isometry of $F$ with the supremum norm onto $C\left(\Omega_{F}\right)$ given by the evaluation mapping. Consider

$$
Z=\left\{x \text { in } X: \Phi^{-1}\left(1_{F}\right)(x)=1\right\}
$$

and define the mapping $\psi: Z \rightarrow \Omega_{F}$ by $\psi(x)=\left.\varepsilon_{x}\right|_{F}$, for each $x$ in $Z$. We claim that $\psi$ is continuous and surjective. Since $\psi$ is a lattice isometry, we have for each $x$ in $Z$,

$$
\left\|\left.\varepsilon_{x}\right|_{F}\right\|=\Phi^{-1}\left(1_{\Omega_{F}}\right)(x)=1 .
$$

It follows that $\psi(x)=\left.\varepsilon_{x}\right|_{F}$ is in $\Omega_{F}$ for each $x$ in $Z$ since each $\psi(x)$ is a real lattice homomorphism on $F$. From the extension lemma it follows that $\psi$ is surjective. By a lemma due to Gleason (see [5], p. 484), it follows that there is a closed subset $Y$ of $Z$ such that $\left.\psi\right|_{Y}$ is a homeomorphism of $Y$ onto $\Omega_{F}$. It is easily seen that the lattice isometry $\left(\left.\psi\right|_{Y}\right)^{0} \circ \Phi$ of $F$ with the supremum norm onto $C(Y)$ is the restriction mapping $R_{Y}$.

The proof of 3.3 will depend upon an extension theorem for lattice homomorphisms into $C(Y)$ for stonian spaces $Y$; the crucial step for that theorem is the extension lemma. The extension theorem was suggested by a theorem of H. P. Lotz (see [9]) on extensions of positive operators; a proof may be given by appropriately modifying Lotz's proof. 
TheORem 3.5. Let $F$ be a Banach lattice, let $Y$ be a stonian space, and let $T: F \rightarrow C(Y)$ be a lattice homomorphism. If $X$ is a compact Hausdorff space such that $C(X)$ contains $F$ as a closed sublattice, then there is an extension $\tilde{T}$ of $T$ that is a lattice homomorphism of $C(X)$ into $C(Y)$. Moreover, $\|\tilde{T}\|=\|T\|$.

Proof of 3.3. We need only show (c) implies (d). If $T$ is a lattice isomorphism of $F$ onto $C(Y)$ for some stonian space $Y$, then there is an extension $\tilde{T}$ of $T$ that is a lattice homomorphism of $C(X)$ onto $C(Y)$ by 3.5. Then $T^{-1} \circ \tilde{T}$ is the desired $\ell$-projection.

Remark. Noting that the extension $\tilde{T}$ constructed in the proof of 3.5 is norm-preserving, we have $\left\|T^{-1} \circ \tilde{T}\right\|=1$ whenever $T$ is an isometry defined on $F$ with the supremum norm. Another proof of 3.1 can be given using this observation.

Proof of Theorem 3.4. The equivalence of (a) and (b) was proved in 3.1. It is clear that (b) implies (c).

If (c) is satisfied, then ex $U_{F^{*}}$ (the set of extreme points of the dual unit ball in $\left.F^{\prime}\right)$ is $\sigma\left(F^{\prime}, F\right)$ closed. It follows from a theorem of Lindenstrauss and Wulbert (see [8], p. 336) that if $F$ is a closed sublattice of $C(X)$, and if ex $U_{F}$. is $\sigma\left(F^{\prime}, F\right)$ closed, then $F$ is isometric to $C\left(\Omega_{F}\right)$ under the evaluation mapping. $\Omega_{F}$ must be stonian since $F$ is order complete. Hence (c) implies (b).

If $\Phi: F \rightarrow C(K)$ is a lattice isometry where $K$ is stonian, then $u=\Phi^{-1}\left(1_{K}\right)$ is a unit element for $F$, and $\left\|\left.\varepsilon_{x}\right|_{F}\right\|=u(x)$ for $x$ in $X$. It follows that (b) implies (d). It is clear that (d) implies (e). If (e) is satisfied, then $\Omega_{F}$ is a continuous image of $Z$ under the mapping $\theta: Z \rightarrow \Omega_{F}, \theta(x)=\left.\varepsilon_{x}\right|_{F}$ (see the proof of 3.1). Hence $\Omega_{F}$ is $\sigma\left(F^{\prime}, F\right)$ compact, and, therefore, $\sigma\left(F^{\prime}, F\right)$ closed. Thus (e) implies (c).

That (b) implies (f) follows since $0 \leqq f$ and $\|f\| \leqq k$ for some positive constant $k$ if and only if $0 \leqq f \leqq k u$, where $u$ is the unit element. If (f) holds, then $u=\sup \{f$ in $F: 0 \leqq f,\|f\| \leqq 1\}$ is a unit for $F$. (b) then follows by the Kakutani representation. The equivalence of (f) and (g) is clear.

To conclude this section, we shall give an example such that the conditions (a) through ( $\mathrm{g}$ ) of 3.4 are not satisfied.

EXAmple 3.6. We give an example of a stonian space $X$ and a closed sublattice $F$ of $C(X)$ such that $F$ is the range of a positive projection on $C(X)$ (so that all of the conditions of 3.3 are satisfied), yet $F$ is not the range of a projection of norm one on $C(X)$ (hence none of the conditions in Theorem 3.4). 
Let $Y$ be an infinite stonian space, and let $y_{0}$ be a nonisolated point of $Y$. Let $F$ be the vector lattice $C(Y)$ with the norm

$$
\|f\|=\sup _{y \in Y}|f(y)| \vee 2\left|f\left(y_{0}\right)\right|, \quad f \text { in } C(Y) .
$$

Then the norm $\|\cdot\|$ on $F$ is equivalent to the supremum norm, and $F$ with the norm $\|\cdot\|$ is an $(A M)$-space. Hence there is a compact Hausdorff space $X$ such that $F$ can be isometrically embedded as a closed sublattice of $C(X)$. It follows from the fact that $Y$ is stonian that there is a positive projection of $C(X)$ onto $F$.

We claim that there is a net of real lattice homomorphisms on $F$, each of norm one, that converges to a real lattice homomorphism on $F$ of norm $\frac{1}{2}$. It will follow that condition (c) of Theorem 3.4 is not satisfied, and hence there is no projection of norm one of $C(X)$ onto $F$. Let $\left\{y_{\alpha}\right\}$ be a net in $Y$ that converges to $y_{0}$, and consider the net $\left\{\varepsilon_{y_{\alpha}}\right\}$ of real lattice homomorphisms on $F$. It is clear that $\left\|\varepsilon_{y_{\alpha}}\right\|=1$ for each $\alpha$, yet $\left\|\varepsilon_{y_{0}}\right\|=$ $\frac{1}{2}$. But $\left\{\varepsilon_{y_{\alpha}}\right\}$ converges to $\varepsilon_{y_{0}}$ in the topology $\sigma\left(F^{\prime}, F\right)$ since $\left\{y_{\alpha}\right\}$ converges to $y_{0}$ in $Y$.

\section{Projections on $C(X)$ for $X$ hyperstonian. A com-} pact Hausdorff space $X$ is called hyperstonian if $X$ is stonian and if the normal measures on $X$ separate the points of $X$. (A measure $\mu$ on $X$ is called normal if $\int f_{\alpha} d \mu \rightarrow 0$ whenever $\left\{f_{\alpha}\right\}$ is a net of nonnegative functions in $C(X)$ such that $\left\{f_{\alpha}\right\}$ decreases to 0 .) Dixmier [3] proved that $X$ is hyperstonian exactly when $C(X)$ is a dual space, and that in this case the predual of $C(X)$ is uniquely determined as the space $C(X)^{n}$ of normal measures on $X$.

We shall consider projections on $C(X)$, for $X$ a hyperstonian space, that satisfy an order continuity condition. We shall call a positive projection $P$ of $C(X)$ onto a sublattice $F$ of $C(X)$ an order continuous projection if $\left\{P\left(f_{\alpha}\right)\right\}$ decreases to 0 in $F$ for each net $\left\{f_{\alpha}\right\}$ in $C(X)$ that decreases to 0 .

A band in an order complete vector lattice $E$ is a lattice ideal $M$ of $E$ such that $\sup S$ is in $M$ for each subset $S$ of $M$ such that $\sup S$ exists in $E$. A simple computation shows that the bands in $C(X)$ for $X$ a stonian space are those lattice ideals hull $(Y)$ such that $Y$ is a closed and open subset of $X$. If $X$ is a hyperstonian space, it can be seen that $P$ is an order continuous $\ell$-projection on $C(X)$ if and only if $\operatorname{Ker} P$ is a band in $C(X)$ (see, for example, [10], Theorem 18.13).

If $Y$ is a subset of $X$, then $-Y$ will denote the set theoretic complement of $Y$.

The situation for order continuous $\ell$-projections, which is partially 
analogous to that for $\ell$-projections on order-complete $C(X)$ spaces, is summarized below.

THEOREM 4.1. Let $X$ be a hyperstonian space and let $F$ be a closed sublattice of $C(X)$. The following assertions are equivalent: $C(X)$.

(a) $F$ is the range of an order continuous $\ell$-projection of norm one on

(b) $F$ is lattice isometric to $C(Y)$ for some closed and open subset $Y$ of $X$. Moreover, the isometry is given by the restriction mapping $R_{Y}$.

(c) $F$ is lattice isometric to a band in $C(X)$.

Proof. If $P$ is an order continuous $\ell$-projection of norm one, then Ker $P$ is a band. It follows that $\operatorname{Ker} P=\operatorname{hull}(Y)$ for some closed and open subset $Y$ of $X$. It follows from 2.4 that $F$ with the supremum norm is lattice isometric to $C(Y)$ under $R_{Y}$.

Suppose that $F$ is lattice isometric to $C(Y)$ under $R_{Y}$ for some closed and open subset $Y$ of $X . \quad C(Y)$ is lattice isometric to hull( $(-Y)$ since $Y$ is both closed and open, and hull $(-Y)$ is a band in $C(X)$.

Suppose that $F$ is lattice isometric to hull $(Z)$ for some closed and open subset $Z$ of $X$. Then $F$ is lattice isometric to $C(-Z)$ under the restriction mapping $R_{-Z}$, and there is an $\ell$-projection of norm one of $C(X)$ onto $F$ with kernel equal to hull $(-Z)$ (see 3.1). Since $Z$ is closed and open in $X$, hull $(-Z)$ is a band in $C(X)$, and the projection is order continuous.

Remark. Using Theorem 3.3 rather than Theorem 3.1, we obtain the following theorem:

THEOREM 4.1'. Let $X$ be a hyperstonian space, and let $F$ be a closed sublattice of $C(X)$. The following assertions are equivalent:

(a) There is an order continuous $\ell$-projection of $C(X)$ onto $F$.

(b) $F$ is lattice isomorphic to a band in $C(X)$.

Notice that $F$ is not necessarily a band. Indeed, if $X$ is a hyperstonian space, and if $Y$ is a proper closed and open subset of $X$, consider the sublattice $F=\left\{f \in C(X): f(y)=\left\{f\left(x_{0}\right)\right\}\right\}$, where $x_{0} \in-Y . \quad F$ is clearly not a band $\left(1_{X} \in F\right)$, yet the mapping $P$ defined by

$$
P(f)(x)=\left\{\begin{array}{ll}
f(x) & x \notin Y \\
f\left(x_{0}\right) & x \in Y
\end{array}, \quad f \in C(X),\right.
$$

is an order continuous $\ell$-projection of $C(X)$ onto $F$.

It is not true that each of the conditions of Theorem 4.1 is equivalent 
to the condition that $F$ be lattice isometric to $C(Y)$ for some hyperstonian space $Y$. For example, let $X$ be a hyperstonian space without isolated points, and let $F$ be the sublattice of $C(X)$ consisting of the constant functions. Then $F$ is lattice isometric to $C$ (one point), yet there is no one-dimensional band in $C(X)$.

Proposition 4.2. Let $X$ be a hyperstonian space, and let $F$ be a closed sublattice of $C(X)$. Then $F$ is lattice isometric to $C(Y)$ for some hyperstonian space $Y$ if and only if $F$ is the range of an order continuous $\ell$-projection of norm one on $C(X)^{\prime \prime}$. Furthermore, each of the equivalent conditions of 4.1 implies that $F$ is lattice isometric to $C(Y)$ for some hyperstonian space $Y$.

Proof. If $F$ is lattice isometric to $C(Y)$ for some hyperstonian space $Y$, then there is an $\ell$-projection $P$ of norm one (but not necessarily order continuous) of $C(X)$ onto $F$. Consider $C(X)^{\prime \prime} \stackrel{P^{\prime \prime}}{\rightarrow} F^{\prime \prime} \stackrel{\varphi^{\prime}}{\rightarrow} F$, where $\varphi$ is the canonical embedding of the predual $F^{n}$ of $F$ into $F^{n^{\prime \prime}}$, and where $P^{\prime \prime}$ is the second adjoint of $P$. Then $\varphi^{\prime} \circ P^{\prime \prime}$ is an order continuous $\ell$-projection of norm one of $C(X)^{\prime \prime}$ onto $F$ (adjoints of positive operators are order continuous).

Conversely, note that $C(X)^{\prime \prime}$ is lattice isometric to $C(Z)$ for some hyperstonian space $Z$. If there is an order continuous $\ell$-projection of norm one of $C(Z)$ onto $F$, then $F$ is lattice isometric to $C(Y)$ via $R_{Y}$ for some closed and open subset $Y$ of $Z$. But a closed and open subset of a hyperstonian space is itself hyperstonian.

As a final remark, we have the following result:

COROLlary 4.3. There is an order continuous $\ell$-projection of $C(X)^{\prime \prime}$ onto $C(X)$ if and only if $X$ is hyperstonian.

This paper constitutes a portion of the author's doctoral thesis at the University of Illinois. The author is grateful to her thesis advisor, Professor Donald R. Sherbert, for his invaluable aid, to Professor Heinrich P. Lotz for his enlightening suggestions, and to the referee for shortening some proofs.

\section{REFERENCES}

1. R. Arens, Projections in continuous function spaces, Duke Math. J., 32 (1965), 469-478.

2. D. W. Dean, Subspaces of $C(H)$ which are direct factors of $C(H)$, Proc. Amer. Math. Soc., 16 (1965), 237-242.

3. J. Dixmier, Sur certains espaces considérés par M. H. Stone, Summa Brasil. Math., 2 (1951), $151-182$. 
4. K. Geba and Z. Semadeni, Spaces of continuous functions V, Studia Math., 19 (1960), 303-320.

5. A. M. Gleason, Projective topological spaces, Illinois J. Math., 2 (1958), 482-489.

6. S. Kakutani, Concrete representations of abstract M-spaces, Ann. of Math., 42 (1941), 994-1024.

7. S. Kaplan, The second dual of the space of continuous functions IV, Trans. Amer. Math. Soc., 113 (1964), 512-546.

8. J. Lindenstrauss and D. Wulbert, On the classification of the Banach spaces whose duals are $L_{1}$ spaces, J. Functional Analysis, 4 (1969), 332-349.

9. H. P. Lotz, Extensions and liftings of positive operators, to appear in Trans. Amer. Math. Soc.

10. W. A. J. Luxemburg and A. C. Zaanen, Riesz Spaces I, North Holland, Amsterdam, 1971.

11. A. L. Peressini, Ordered Topological Vector Spaces, Harper and Row, New York, 1967.

12. H. H. Schaefer, Topological Vector Spaces, Macmillan, New York, 1966.

13. D. Wulbert, Averaging projections, Illinois J. Math., 13 (1969), 689-693.

14. - Some complemented subspaces of $C(X)$, Pacific J. Math., 24 (1968), 589-602.

Received March 10, 1976. Most of the results of this paper were obtained while the author was supported by a National Science Foundation Graduate Fellowship.

UNIVERSITY OF ILLINOIS - URBANA 


\section{PACIFIC JOURNAL OF MATHEMATICS}

\section{EDITORS}

RICHARI) ARENS (Managing Editor)

University of California

Los Angeles, CA 90024

R. A. Beaumiont

University of Washington

Seattle, WA 98105

\section{J. DugunduI}

Department of Mathematics University of Southern California Los Angeles, CA 90007

D. Gilbarg and J. Milgram Stanford University

Stanford, CA 94305

\section{ASSOCIATE EDITORS}

E. F. BECKENBACH
B. H. NEUMANN

F. Wolf

K. YoshidA

\section{SUPPORTING INSTITUTIONS}

UNIVERSITY OF BRITISH COLUMBIA CALIFORNIA INSTITUTE OF TECHNOLOGY

UNIVERSITY OF CALIFORNIA

MONTANA STATE UNIVERSITY

UNIVERSITY OF NEVADA

NEW MEXICO STATE UNIVERSITY

OREGON STATE UNIVERSITY

UNIVERSITY OF OREGON

OSAKA UNIVERSITY

\author{
UNIVERSITY OF SOUTHERN CALIFORNIA \\ STANFORD UNIVERSITY \\ UNIVERSITY OF HAWAII \\ UNIVERSITY OF TOKYO \\ UNIVERSITY OF UTAH \\ WASHINGTON STATE UNIVERSITY \\ UNIVERSITY OF WASHINGTON \\ AMERICAN MATHEMATICAL SOCIETY
}

The Supporting Institutions listed above contribute to the cost of publication of this Journal, but they are not owners or publishers and have no responsibility for its contents or policies.

Mathematical papers intended for publication in the Pacific Journal of Mathematics should be in typed form or offset-reproduced (not dittoed), double spaced with large margins. Underline Greek letters in red, German in green, and script in blue. The first $p: 1<$ graph or two must be capable of being used separately as a synopsis of the entire paper. Items of the biblography should not be cited there unless absolutely necessary, in which case they must he identified by author and Journal, rather than by item number. Manuscripts, in duplicate, may be sent to any one of the four editors. Please classify according to the scheme of Math. Reviews, Index to Vol. 39. All other communications should be addressed to the managing editor, or Elaine Barth, University of California, Los Angeles, California, 90024.

100 reprints are provided free for each article, only if page charges have been substantially paid. Additional copies may be obtained at cost in multiples of 50 .

The Pacific Journal of Mathematics is issued monthly as of January 1966. Regular subscription rate: $\$ 72.00$ a year (6 Vols., 12 issues). Special rate: $\$ 36.00$ a year to individual members of supporting institutions.

Subscriptions, orders for back numbers, and changes of address should be sent to Pacific Journal of Mathematics, 103 Highland Boulevard, Berkeley, California, 94708.

PUBLISHED BY PACIFIC JOURNAL OF MATHEMATICS, A NON-PROFIT CORPORATION Printed at Jerusalem Academic Press, POB 2390, Jerusalem, Israel.

\section{Copyright (C) 1976 Pacific Journal of Mathematics} All Rights Reserved 


\section{Pacific Journal of Mathematics}

\section{Vol. 66, No. 2 December, 1976}

Gerald A. Beer, Tax structures whose progressivity is inflation neutral..... 305

William M. Cornette, A generalization of the unit interval............. 313

David E. Evans, Unbounded completely positive linear maps on

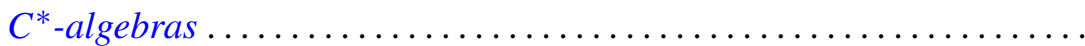

Hector O. Fattorini, Some remarks on convolution equations for

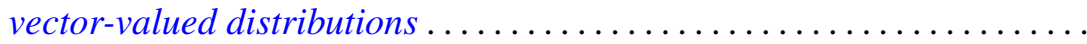

Amassa Courtney Fauntleroy, Automorphism groups of unipotent groups of

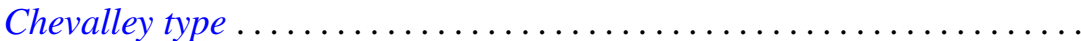

Christian C. Fenske and Heinz-Otto Peitgen, On fixed points of zero index in

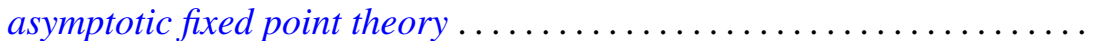

Atsushi Inoue, On a class of unbounded operator algebras. II ............

Herbert Meyer Kamowitz, The spectra of endomorphisms of algebras of

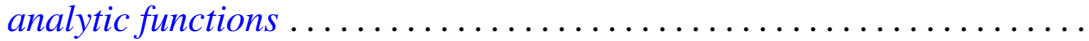

Jimmie Don Lawson, Embeddings of compact convex sets and locally compact cones ....................................

William Lindgren and Peter Joseph Nyikos, Spaces with bases satisfying certain order and intersection properties .....................

Emily Mann Peck, Lattice projections on continuous function spaces ...... 477

Morris Marden and Peter A. McCoy, Level sets of polynomials in $n$ real variables...

Francis Joseph Narcowich, An imbedding theorem for indeterminate Hermitian moment sequences......................

John Dacey O'Neill, Rings whose additive subgroups are subrings ...

Chull Park and David Lee Skoug, Wiener integrals over the sets bounded by sectionally continuous barriers .....................

Vladimir Scheffer, Partial regularity of solutions to the Navier-Stokes equations.

Eugene Spiegel and Allan Trojan, On semi-simple group algebras. II

Katsuo Takano, On Cameron and Storvick's operator valued function space integral 